\title{
Using Robotics and A.I. to Physically Explore a Space of Aesthetic Possibilities
}

\author{
Defining a Physical Aesthetic Experience by the Targeted EEG Feedback of the Perceiver
}

Emanuel Gollob

Creative Robotics, UFG Linz

Linz, Upper Austria, Austria

emanuel@emanuelgollob.com

\author{
Magdalena Mayer \\ University of Vienna \\ Vienna, Vienna, Austria \\ magdalena.mayer@salzburg.co.at
}

\author{
Johannes Braumann \\ Creative Robotics, UFG Linz \\ Linz, Upper Austria, Austria \\ johannes@robotsinarchitecture.org
}

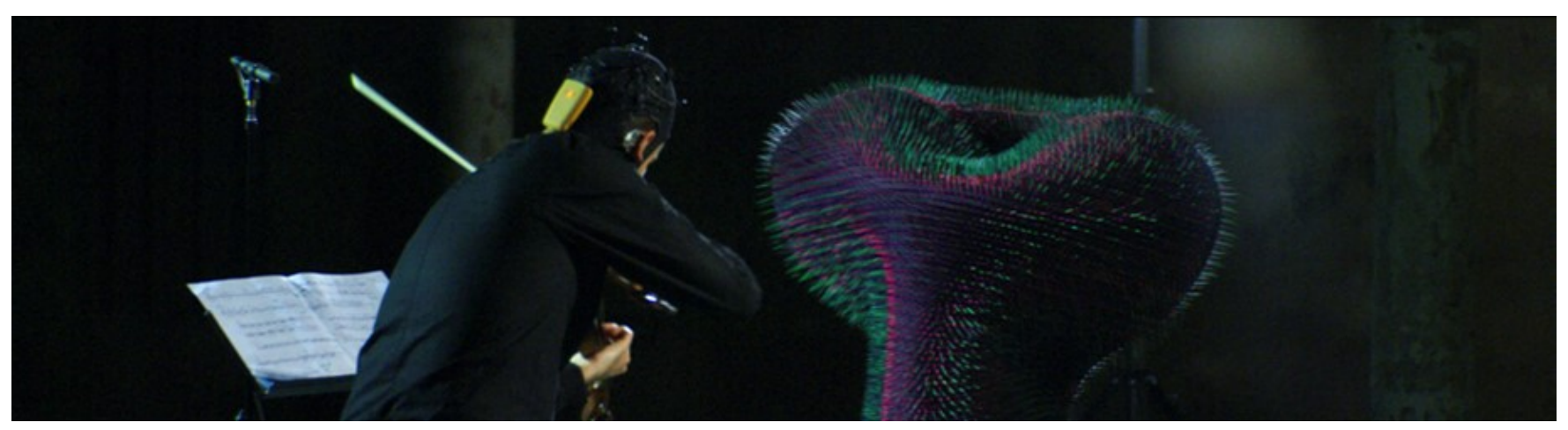

Figure 1: Violin player improvising a modular composition while being connected with Doing Nothing with AI 1.0

\begin{abstract}
Aesthetic perception and cognition processes are highly individual dynamic processes due to their dependency on the emotional affective state, perceptual analysis, memory, context, and cognitive mastering. Therefore, an aesthetic experience will always be perceived differently for every person and time. With our approach, we research the potential to define an adaptive physical aesthetic experience by the targeted EEG feedback of the perceiver.

In a series of three distinct projects, we use generative robotic control (KUKA|prc), a Deep Convolutional Generative Adversarial Network, and electroencephalography (EEG) to create an aesthetic adaptation strategy within a physical parametric output space.

Though certain physical limitations apply, the resulting artefact interaction offers the potential to make the aesthetic definition to a certain extend relational to individual perception and cognition processes and, therefore, to some extent adaptive to emotional, contextual, and cultural change over time.
\end{abstract}

\section{CCS CONCEPTS}

- Human-centered computing $\rightarrow$ Interaction design theory, concepts and paradigms; $\bullet$ Applied computing $\rightarrow$ Arts and humanities; • Computing methodologies $\rightarrow$ Artificial intelligence.

Permission to make digital or hard copies of part or all of this work for personal or classroom use is granted without fee provided that copies are not made or distributed for profit or commercial advantage and that copies bear this notice and the full citation on the first page. Copyrights for third-party components of this work must be honored.

For all other uses, contact the owner/author(s).

TEI '21, February 14-17, 2021, Salzburg, Austria

(C) 2021 Copyright held by the owner/author(s).

ACM ISBN 978-1-4503-8213-7/21/02.

https://doi.org/10.1145/3430524.3440647

\section{KEYWORDS}

parametric robot control, generative aesthetics, neuroaesthetics, generative adversarial network, electroencephalography

\section{ACM Reference Format:}

Emanuel Gollob, Magdalena Mayer, and Johannes Braumann. 2021. Using Robotics and A.I. to Physically Explore a Space of Aesthetic Possibilities: Defining a Physical Aesthetic Experience by the Targeted EEG Feedback of the Perceiver. In Fifteenth International Conference on Tangible, Embedded, and Embodied Interaction (TEI '21), February 14-17, 2021, Salzburg, Austria. ACM, New York, NY, USA, 8 pages. https://doi.org/10.1145/3430524.3440647

\section{INTRODUCTION}

Imagine two persons in an exhibition looking at the same sculpture. Even if sculpture, context, and time are the same, both will likely develop very different aesthetic judgments and aesthetic emotions [21]. In the last decade, empirical aesthetics [4, 22, 29] and neuroaesthetics [28] have made significant progress in mapping the components involved in the process of aesthetic perception and cognition. But what if it was the other way around, and we have the sculpture reacting to the aesthetic judgment and emotion of the perceiver targeting a very specific neuronal outcome?

This paper presents three different prototypical, neuro-reactive installations with an embedded aesthetic strategy targeting a specific electroencephalography (EEG) feedback. All utilize generative robotic control and deep convolutional generative adversarial networks to adapt their different media based on the electroencephalography (EEG) feedback of the perceiver.

With these artefacts, we aim to research the potential to define a physical adaptive aesthetic experience by the targeted EEG feedback of the perceiver. With our work, we on purpose bridge 
various disciplines and, by doing so, also hope to contribute to topics relevant in each of the disciplines and help shape a common ground between those disciplines. For example, with this explorative study on an embedded aesthetic human-machine-interaction, we also hope to contribute to the neuroaesthetics topic of bottomup perception as well as to foster knowledge about the potential of utilizing EEG-feed generative adversarial networks for interactive art.

\section{THEORETICAL BACKGROUND}

Aesthetic perception and cognition are a complex interplay of a variety of components. Besides context and object features, there are many more dimensions that influence an aesthetic experience. This complex relationship is best described in the model of aesthetic experience by Leder et al. [22]. According to this model, aesthetic experience can be split into five subprocesses: perceptional analysis, memory integration, explicit classification, cognitive mastering, and evaluation. The model suggests two types of outputs, aesthetic judgment and aesthetic emotion. The aesthetic judgment is connected to prior knowledge as well as individual expectations. The emotional state rises from the affective state and a continuous feedback loop between evaluation and cognitive processes. So, aesthetic experience as a whole is a very dynamic process.

While human brains are specialized for complex and dynamic real-world tasks, as aesthetic perception, most neuroscientific studies take place in highly controlled laboratory settings to ensure science criteria. This reduction in environmental complexity, however, also limits the range of behaviors that can be explored. Only a few explorative studies are carried out on a large scale within complex natural environments [19].

However, machine learning has shown to be capable of solving complex problems in various sciences [27]. The advantage of artificial neural networks is their data processing characteristics. Artificial neural networks are tolerant of diverse data and have high learning and generalization abilities [25]. In 2014, Goodfellow et al. proposed the artificial neural network type Generative Adversarial Networks (GANs) [14]. These networks are especially suited for generating $2 \mathrm{D}$ visual content [33] but can also be applied to 3D object generation $[9,32]$ and various other tasks. There are a few advantages of GANs over other neural networks: First, GANs can generate data that is indistinguishable from real-world data. Second, the GAN generator trains a mapping from a low-dimensional probabilistic space to the output space. Once trained, this enables the generator to generate samples without further feedback. Third, the GAN discriminator learns to imitate the dataset labeling process and becomes a classifier [32].

In the rapidly developing field of neuroaesthetics, studies have successfully shown that the dynamics of aesthetic experience can be correlated with brain activity measurable with fMRI [2] as well as with electroencephalography [31].

Due to our need to react to EEG data in near real-time, we also build on Brain-Computer Interaction knowledge. Research in this field can usually be divided into: Active, Reactive, and Passive [16] Matthew Pike et al. explored a fourth concept named affect loop [18]. In an affect loop, a user is affected by a system that, in turn, is affected by the user. The project "\#Scanners: Exploring the control of adaptive films using brain-computer interaction "by Matthew Pike et al. [30] is one example of an affect loop. In this project, Matthew Pike et al. used a Brain-Computer Interaction for a 2D bio-responsive entertainment.

In comparison, parametric architecture and design have a long tradition in using generative design and algorithms to define 3D physical aesthetic experiences. Parametric design usually builds on evolutionary algorithms with either defined single or multiple objectives or guided by choice [17]. However, parametric architecture and design merely focus on finding an ideal static aesthetic for a specific target group, context, and time [3]. Adapting a generative aesthetic to a dynamic objective, like EEG feedback certainly is, however, demands an aesthetic in flux.

Within the context of physical adaptive generative aesthetics, it is essential to respond to each action with an appropriate physical, in this case, machinic reaction. We utilize robotic arms because they provide a highly robust, accurate, and fast platform for machinic motion without requiring a specific mechatronic skillset towards developing custom machines.

However, due to the software architecture of industrial robots, realizing real-time control can be challenging. While a robotic fabrication line may seem like it is orchestrated in real-time, with all its robots working in perfect synchronicity, it consists mostly of preprogrammed movements triggered by an industrial programmable logic controller.

"True" real-time control of a robot is achieved by providing a set value for the robot's position, deterministically at the robot's cycle time of usually $12 \mathrm{~ms}$ (referred to as hard-real time). However, the required deterministic behavior of software cannot be reliably achieved in standard desktop operating systems. In contrast, it is possible to control a robot through non-real-time interfaces [6, 7] so it requires user-specific programming rather than a standardized API. More recently, KUKA presented mxAutomation as a way to allow PLCs to control robotic arms without requiring the programmer to have robot-programming knowledge. This functionality can also be accessed from languages like $\mathrm{C}$ and $\mathrm{C \#}$ and, in practice, comes close to what one could consider an API.

mxAutomation implements a buffer that can compensate for timeouts, thus resolving the challenge of providing data in hard real-time. As a robot control environment, we utilize Grasshopper, a visual programming language for Rhino3D. Grasshopper is deeply linked with generative design. Its combination with Rhino3D provides an easy to use environment for simulations and generative control. To realize a direct interaction between Grasshopper and mxAutomation, it was necessary to define a data-flow strategy that considers Grasshopper's acyclic behavior and constantly provides updated motion commands to the robotic manipulator.

In this exploratory research, we combine all four of the above elements (aesthetic research, BCI, generative machine learning, and parametric robotic control) to look into the potential to define an adaptive physical aesthetic experience by the targeted EEG feedback of the perceiver. We explore the use of Generative Adversarial Networks to adapt the parameters of a bottom-up perception process. 


\section{$2.1 \quad$ Related Works}

This paper can be situated in the wider context of aesthetic experience, brain-machine interfaces, and interactive robotics. There are a hand-full of projects that look into similar topics:

One is, for example, "My Virtual Dream": Collective Neurofeedback in an Immersive Art Environment [19], a study collecting EEG data from 523 participants during a night-long collective computer game. In the game, each of the participants had the opportunity to manipulate his mental state of concentration and relaxation. The project not only shows insights on the training effects in natural environments but also shows the potential of bridging art and neuroscience.

In interactive robotics, we want to mention Gannon's and Sandoval's work "Manus" [10]. It consists of ten industrial robots that collectively interact with people passing by. Gannon and Sandoval show in their work the potentials of robotic behavior as a language to communicate and connect with humans. To a certain extent, we continue this direction. Instead of defining a language, we set up a learning system with a space of possible actions and an embedded goal.

In their work Double-Taker (Snout) [23] Golan Levin et al. explore beside others subjecthood and gestural choreographic interaction based on real-time machine vision. With our work, we extend on this by combing gestural choreography with non-hierarchical interaction and EEG feedback.

In the intersection of $\mathrm{BCI}$ and bio-responsive film, the project "\#Scanners: Exploring the control of adaptive films using braincomputer interaction" [30] by Matthew Pike et al. was a great inspiration for us. It explores the use of BCI in forms of partiallyinvoluntary and partially conscious control of the film. With the use of a GAN as an interaction mediator in our artifacts and the possibility of targeting unconscious EEG feedback, we also situate ourselves between the unconscious and conscious BCI control. We intend to expand on this by adding a physical dimension to the responsive media.

We share the ambition of designing a skin for nonverbal communication with Caress of the Gaze and Synapse [8] by Farahi. Compared to Farahi, we are not so much interested in visualizing the state of the wearer's mind but rather aiming at studying nonhierarchical interactions, in which the skin is in dialogue with its interactant.

\subsection{Method}

The artefacts constructed for this paper are a neuro-reactive robotic installation consisting of an EEG device being connected with a KUKA industrial robotic arm, a Generative Adversarial Network, and a database. Together forming an affect loop [18] in which the EEG wearing spectator shows a reaction to a physical aesthetic experience that at the same time is adaptive to the user's EEG feedback.

For public exhibitions, we use a Muse 2016 EEG headband due to its universal head size fit and easy handling. We apply a Fast Fourier Transform (FFT) method on the raw EEG data stream.

For our specific use case targeting default-mode brain network activity [15] we use the relative spectral power in alpha and beta frequencies as an indicator for concentration [19]. We then use concentration as an indicator of cognitive task activity and cognitive task activity as a negative indicator for the likelihood of defaultmode brain network activity.

For more scientific settings, we use an Enobio 8 EEG cap from Neuroelectrics with eight electrodes. The raw EEG is streamed via a lab streaming layer to an EEG processing pipeline. The EEG processing pipeline applies a Fast Fourier Transform method and Loretta source localization according to the regions of interest (ROIs) and frequency bands specified as statistical correlation with the DMN signal by Irene Neuner et al. [26]. The Enobio 8 EEG cap has eight channels with a sampling rate of $500 \mathrm{~Hz}$ compared to the Muse 2016 EEG headband, which has four electrodes and a sampling rate of $255 \mathrm{~Hz}$. The electrode positions we use with the EEG cap are visualized in Figure 2.

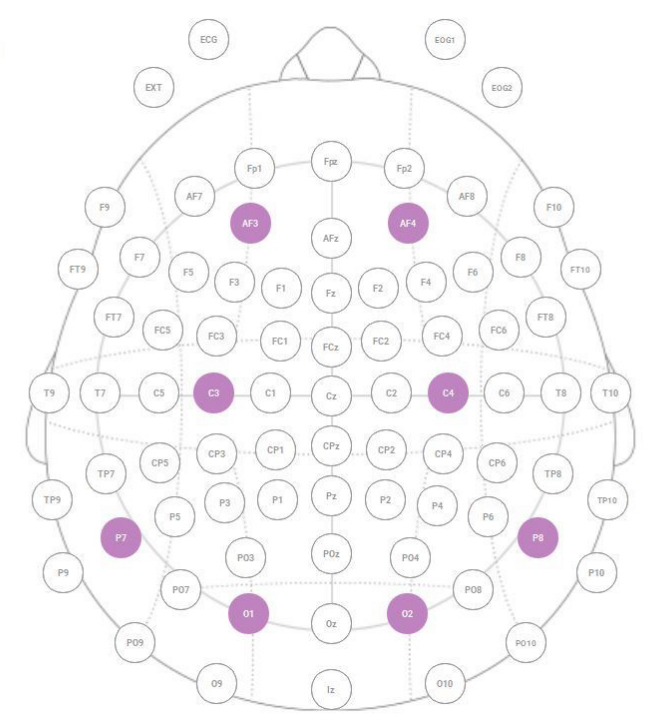

Figure 2: Electrode positions according to the 10-20 system

For generating the new aesthetic parameters and learning from the EEG feedback, we use a Deep Convolutional Generative Adversarial Network (DCGAN) with a Tensorflow backend in Python 3.7. Its generator sequentially tries to come up with new aesthetic parameter samples. Its discriminator sequentially trains itself on the database of aesthetic parameter samples with positive EEG feedback. Whenever the robotic system demands a new parameter sample, the latest generated is executed physically (Figure 3). For every exhibition, a new GAN machine learning model is created.

In all three projects, we use a KUKA KR6 R900 industrial robot with a KRC4 controller and mxAutomation. The KUKA KR6 R900 robot has six axes of movement and a maximum reach of $900 \mathrm{~mm}$. As generative robotic control software, we use KUKA|prc [5], a plugin for Grasshopper within Rhino3D. To parameterize the robotic solution space for our projects, we define that all axis motions have to be described as an array of sine waves. Each of the parameters defining the sine waves is then again defined by a sine wave. On the one hand, this is due to the demand for making choreographies describable while, on the other hand, following our 


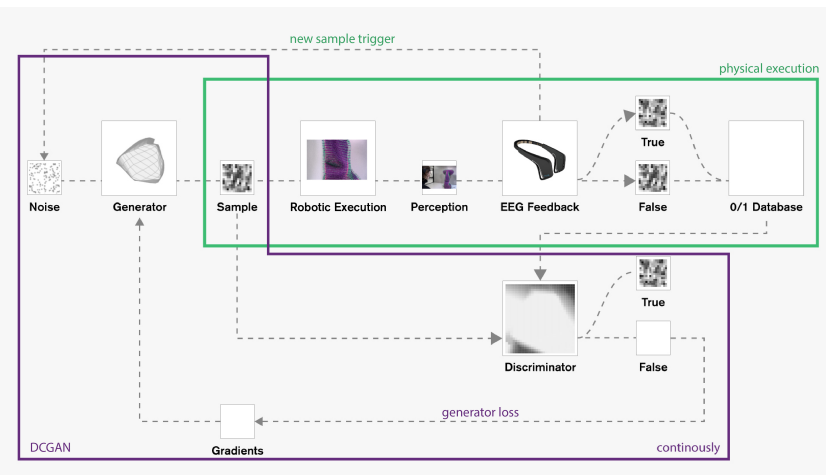

Figure 3: Adapted DCGAN Setup

use case demand of fluid motions and changeability of rhythm and narration.

The database is filled up during the exhibitions with positive and negative evaluated parameter samples. Each sample consists of 256 values in the range of $0-255$. As we have 256 aesthetic parameters with 255 steps each, the theoretical number of possibilities is 255 by the power of 256 . In practice, human perception is not able to perceive each parameter difference. Making the parameter space translatable to a $16 \times 16$ greyscale image with 256 pixels (Figure 4) creates the advantage of using $2 \mathrm{D} \mathrm{CNN}$ with a fast learning rate but the trade-off of parameter abstraction.

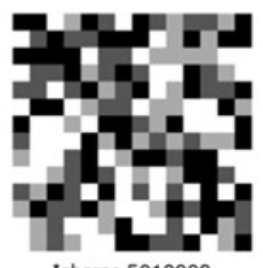

"choreo 5012902

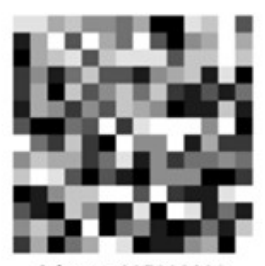

"choreo 897908890

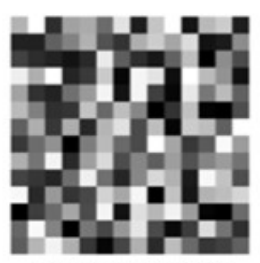

"choreo 378906323
Figure 4: Parameter samples translated to $16 \times 16$ greyscale images

In all three projects, we use the visual programming software $v v v v$ gamma as an interface between EEG feedback, database, GAN setup, and the robotic control software KUKA|prc. We add up the EEG feedback in $v v v v$ gamma until our specified threshold of either positive or negative evaluation is reached. If the evaluation is positive according to our targeted EEG feedback, then the parameter sample is saved as "True" and the next parameter sample is going to be only slightly modified. If the feedback is negative, a new generator sample is executed physically.

\section{PROJECTS}

Over one year, we developed three different prototypical projects at the intersection of aesthetic research, neuroscience, and machine learning, each focusing on a particular aspect and providing various levels of interactivity.

- "Doing Nothing with AI 1.0" is a robotic art installation that uses generative robotic control, EEG measurements, and a
DCGAN machine learning model to optimize its parametric movement.

- "Doing Nothing with AI 2.0" builds on "Doing Nothing with AI 1.0" and extends its aesthetic space of possibilities with parametric sound and parametric visuals.

- A third project is similar to "Doing Nothing with AI 1.0". However, the perceiver is improvising a violin play inspired by his perception while being connected to the installation.

\subsection{Doing Nothing with AI 1.0}

"Doing Nothing with AI 1.0" is a robotic art installation that uses generative robotic control, EEG measurements, and a DCGAN machine learning model to optimize its parametric choreography with the aim to activate the spectator's default-mode brain network [2, 26] (Figure 5).

We target the default-mode brain network activity as Belfi et al. [2] found correlations between the dynamics of aesthetic experience and default-mode network activity. Default-mode brain network activity is an unconscious brain activity distributed across several regions in our brain. This brain network tends to show less activity when we are actively pursuing a task or concentrating on something and higher activity if we do not engage in any particular mental tasks.

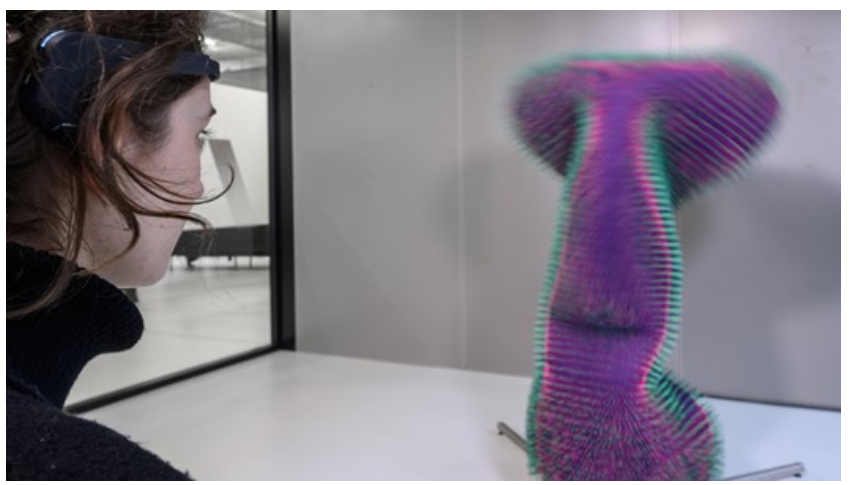

Figure 5: Doing Nothing with AI 1.0. Video is available online [11]

The parametric solution space is framed by limitations of the skin material, the ergonomics of the robot, and our rule of fluid motions. Within this solution space, the installation continuously tries to find a parameter combination that supports the spectator's default-mode brain network activity.

For this iteration, we defined that for each of the six axes, the motion range, speed, rhythm, and narration are mutable parameters. We further targeted a certain visual attraction and familiarity [20] with other natural and digital phenomena as shared features (e.g., sea anemones and coral structures as well as digitally animated endless videos). Familiarity increases visual attraction as well as decreases the alien effect of unknown moving artefacts [20]. Additionally, we integrated the concept of humans preferring curved visual objects [1].

We further decided to costume the robot entirely to conceal the potentially threatening element of industrial machinery. 
3.1.1 Design rationale. Our design decisions on skin materiality, coloring, and shape were guided by our aim to amplify the robot's slightest movement change and make the output space more distinguishable.

The skin of "Doing Nothing with AI 1.0" is fabricated out of 23.000 toothpicks and foam. Each toothpick has a two-colored top and a single-colored bottom. All toothpicks are placed at a distance of $12 \mathrm{~mm}$ to each other. Every second toothpick line is shifted $6 \mathrm{~mm}$, and $180^{\circ}$ rotated. This creates a surface that uses toothpicks as surface-normals to amplify the local surface change and color effects to underline the overall motion. The decision upon the shape was a reaction to the robot ergonomic and motion features and again pursuing the aim to amplify the robot's movements. For example, the oval-shaped top increases the movement visibility of the last two robot axes (A5 and A6).

The skin was made in a co-production process with the robot. The robot picked, glued, and placed the toothpicks itself.

In this iteration, sound is not our field of interest. Still, whenever a toothpick is touching another, it creates a soft noise adding up to the robot soundscape. Both soundscapes together create an acoustic atmosphere that underlines the visual experience. Smell and touch were not designed in this iteration.

3.1.2 Procedure. Spectators approaching the installation are introduced to the project by a staff member. The staff member supports the spectators in the EEG device placement. Once the electrode connection is established, the installation starts automatically. The first aesthetic parameters will be generated based on the collected feedback of previous users. In a time ranging from 30-60 seconds, the first evaluation threshold is achieved. Suppose the evaluation is positive according to our targeted EEG feedback. The parameter sample is saved as "True" and the next parameter sample will only be slightly modified. If the feedback is negative according to our targeted EEG feedback, a new generator sample is executed physically. The spectator has the free choice to continue or stop the interaction at any time. Once the spectator decides to take off the EEG device, the installation automatically switches to standby mode.

3.1.3 Specific learnings. For perceivers untrained to movement nuances, it can be hard to recognize small parameter changes in movements.

Although the skin material of Doing Nothing with AI 1.0 was developed to allow a large space of possible color effects and motions, its fragile materiality limited the possibilities.

Also, it can be noted that several spectators wanted to touch the skin and, on their first impression, expected it to be soft.

\subsection{Doing Nothing with AI 2.0}

"Doing Nothing with AI 2.0" builds on the experience of "Doing Nothing with AI 1.0" and extends its aesthetic space of possibilities with parametric sound and parametric visuals (Figure 6).

The parametric visuals are generated by translating the current aesthetic parameters to visuals in Max/MSP and Modul8. The visuals are then projected with a laser projector on the translucent robot skin, floor, and the visitor. The parametric soundscape is generated by translating the current aesthetic parameters to 4 channel surround sound in Max/MSP. One bass and four head speakers were placed in the four corners of the room. In this project, the setting emphasized a spatial aesthetic experience and invited the visitor to move in it.

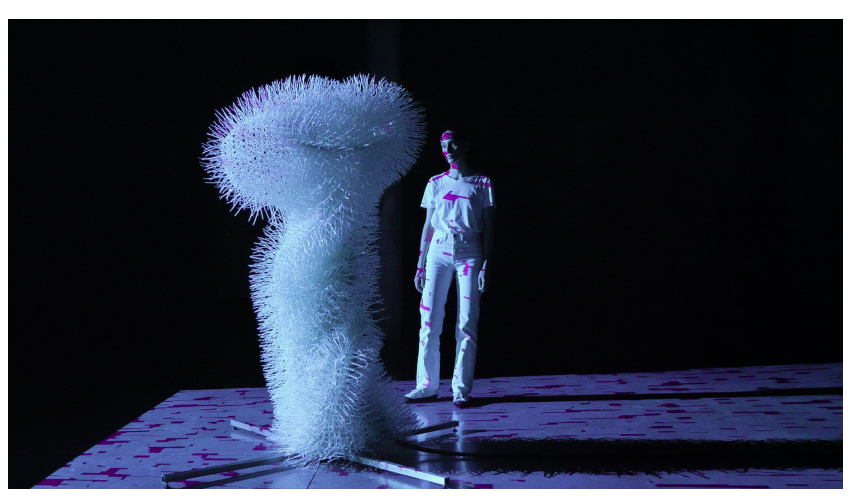

Figure 6: Doing Nothing with AI 2.0. Video is available online [12]

3.2.1 Design rationale. Based on our experience that a certain number of spectators have difficulties distinguishing small changes in movement, we, for this iteration, focused on extending the space of aesthetic possibilities with parametric sound and visuals. Additionally, we wanted to decrease the influence of the context by creating an immersive experience.

To do so, we fabricated a robot skin out of approximately 25.000 cable ties. We used cable ties as skin material as it allows us to create a translucent 3D surface that we can project on. At the same time, the durability of cable ties allows a wider space of possible robot motions. For being able to project a wider color space, we use a laser projector. The decisions upon which visual and sound parameters we treat as variables and which ones as static shared features were not predefined but developed within the prototyping process.

As in the previous iteration, the robot costume form's decision was again a reaction to the robot's ergonomic and motion features.

Also, in this iteration, smell and touch were not part of our design decisions.

3.2.2 Procedure. The procedure is the same as in "Doing Nothing with AI 1.0" with the difference that the visitor can move freely.

3.2.3 Specific learnings. Compared to creating a high dimensional aesthetic space of possibilities in a single media (robotic movement), the creation of a high dimensional aesthetic space of possibilities bridging different media (movement, sound, and visuals) demanded an intense cooperation process to achieve the impression of a homogenous aesthetic experience rather than three contradicting media experiences. Besides the extraction of possible parameters in all three media and the relational tuning of each parameter range, this process also involved a definition and review loop of which aesthetic parameters we address as additional dimensions and which ones we relate to a parent parameter (e.g., sound direction and robot movement direction). 


\subsection{Violin improvisation \& Doing Nothing with AI 1.0}

This project setup is similar to the setup of "Doing Nothing with AI 1.0". The difference is that the perceiver is improvising a violin play inspired by his perception while being connected to the installation (Figure 1 and Figure 7). This, on the one hand, might make it impossible for the installation to keep the violin player in the default-mode while, on the other hand, creates a beautiful dialogue of two actors desperately trying to fulfill their embedded aims. The violin player improvises a modular music piece consisting of 10 modules. Each module has a length between 10-30 seconds, can be combined with the other modules in any order and can be played in different stroke intensities and several times.

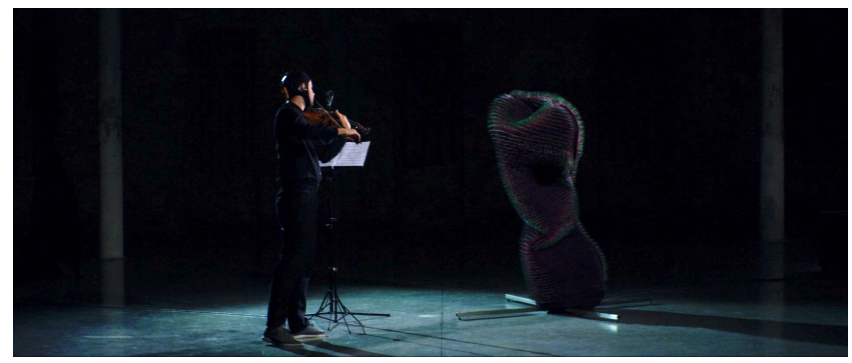

Figure 7: Violin player improvising a modular violin composition while being connected with Doing Nothing with AI 1.0. Video is available online [13]

3.3.1 Design rationale. All costume design decisions were the same as in "Doing Nothing with AI 1.0".

3.3.2 Procedure. For this project, the violin player wears the Enobio 8 EEG cap with eight electrodes, and we use the Loretta source localization with the ROIs, according to Irene Neuner et al. [26]. The installation and the violin player are positioned in opposition to each other 2 meters apart. The robot starts to move in the dark, and with increasing lightning, the violin player starts to improvise the violin play. Over 10 minutes, the violin player improvises based on his aesthetic experience, and the DCGAN evolves the installations physically executed aesthetic parameters according to the player's EEG feedback.

3.3.3 Specific learnings. One interesting dimension to point out would be the extent to which the installation's aesthetic appearance depends on the interactants, meaning the perceiver and the robotic sculpture itself. Regarding the performance that took place with an audience watching, usually, an artist might want to have control over the actual aesthetic of his piece and the choreography performed. However, in this case, not the aesthetic decision itself is defined by us, but rather a behavioral pattern behind it. We were aware that this means a loss of control concerning the actual choreography, while also expecting the possibility of an even more authentic and richer, because reactive, aesthetic experience.

Even spectators with prior knowledge about the role of the EEG feedback explained a feeling of satisfaction every time it seemed that the movement fits the music or the other way around.

\section{RESULTS AND LEARNINGS}

Though certain physical limitations apply, we have observed that the resulting artefact interaction offers the potential to make the aesthetic definition, to a certain extent, relational to individual perception and cognition processes. Therefore, to some extent, it can be understood as adapting to emotional, contextual, and cultural change over time.

Throughout all our projects, we have not saved the EEG data itself, but only the positive and negative evaluated aesthetic parameters. As several components in our exploratory research were not standardized, we want to clearly distance ourselves from interpreting this data and only share our observations and experiences in these three projects.

As our adaptive aesthetic strategy demands an aesthetic in the flux, the resulting physical aesthetic experience is not just an optimized static aesthetic but rather an embedded aesthetic interaction. None of the interactants controls the other, but both entities act, perceive, learn, and react in a non-hierarchical, one could say dialogue-like setting.

Although we, in general, favor explanatory algorithms and transparency, we experienced that we have to hide the technological layers in favor of the affect loop interaction as much as possible. Still, a few components as the EEG devices or the safety system could not be hidden. We observed that every visible technical device or logic easily dragged attention to its technical functions rather than the interaction with the aesthetic and the conceptual thoughts behind it.

Finally, we also observed that to create an embedded aesthetic interaction affect loop in a real-world setting, it was very beneficial to the interaction that we targeted an unconscious brain network as this very much limited the potential misuse as a BCI control device.

\section{DISCUSSION}

We see this paper as a general approach to defining an adaptive physical aesthetic experience by any targeted EEG feedback. We targeted default-mode network brain activity in our projects as it was in line with our conceptual interest. Still, e.g., in case some would aim to create an aesthetic experience that targets different EEG feedback, our approach could be easily adapted. Regarding the unstandardized setting within which we conducted our exploratory research, there are, of course, limitations that have to be taken into account.

A limitation and benefit at the same time is the stochastic nature of GAN machine learning. This means that the outcome of the method proposed in this paper will be different every time. It is important to highlight that GANs are known as black-box algorithms and most challenging to understand by machine learning experts and specialists in the application area [24].

As our projects were only shown interactively in the context of media and technology art events, we assume mainly visitors with a high interest in either art or technology or both were interacting with our systems. Due to this selection bias, it would be highly interesting to conduct an experiment with a more diverse sample.

The decision to use a KUKA industrial robot as a parametric motion tool allowed us to embed near real-time interaction with 
industrial precession but also very much defined and therefore restricted the space of aesthetic possibilities we could research.

For further research, we would emphasize to consider explainable learning systems instead of black-boxes and suggest a more diverse range of participants and real-world contexts.

Parts of this explorative research were inspired by our interest in understanding the influence of living in a digital culture on our default-mode brain network activations and understanding how perception and brain processes might be shifting parallel to digitalization. In line with our interest, we propose considering such a system to be long-term explorative research, collecting aesthetic feedback periodically over years or even decades.

\section{ACKNOWLEDGMENTS}

This project has received funding from MindSpaces under Grant No.: 825079 (https://mindspaces.eu/). MindSpaces is a 3-year research project financed by the European Union's Horizon 2020 program in the framework of STARTS initiative.

"Doing Nothing with AI 1.0" and "Doing Nothing with AI 2.0" were supported by Vienna Business Agency.

Parts of "Doing Nothing with AI 1.0" were produced at Design Investigations studio at the University of Applied Arts Vienna. Veronika Mayer created the parametric soundscape of "Doing Nothing with AI 2.0". Conny Zenk made the parametric visual art of "Doing Nothing with AI 2.0".

The real name of "Violin improvisation \& Doing Nothing with AI 1.0 " is "Shaky Savine \& Doing Nothing with AI 1.0". Armin Sanayei is the composer of the violin improvisation "Shaky Savine", which was improvised by Jacobo Hernández Enríquez. "Shaky Savine \& Doing Nothing with AI 1.0" was co-produced by REAKTOR \& Reconsil and documented by Anna Mitterer, Philipp WindsorTopolsky, Tobias Aschermann, Aram Baronian and Alexander Yannilos.

Published as Open Access with the support of the University of Art and Design Linz.

\section{REFERENCES}

[1] Moshe Bar and Maital Neta. 2006. Humans prefer curved visual objects. Psychological Science 17, 8 (2006), 645-648. https://doi.org/10.1111/j.1467-9280.2006. 01759.x

[2] Amy M. Belfi, Edward A. Vessel, Aenne Brielmann, Ayse Ilkay Isik, Anjan Chatterjee, Helmut Leder, Denis G. Pelli, and G. Gabrielle Starr. 2019. Dynamics of aes thetic experience are reflected in the default-mode network. NeuroImage 188 , November 2018 (2019), 584-597. https://doi.org/10.1016/j.neuroimage.2018.12.017

[3] Shajay Bhooshan. 2017. Parametric design thinking: A case-study of practiceembedded architectural research. Design Studies 52 (2017), 115-143. https: //doi.org/10.1016/j.destud.2017.05.003

[4] Janneke Blijlevens, Paul Hekkert, Helmut Leder, Clementine Thurgood, Lin Lin Chen, and T. W.Allan Whitfield. 2017. The aesthetic pleasure in design scale: The development of a scale to measure aesthetic pleasure for designed artifacts. Psychology of Aesthetics, Creativity, and the Arts 11, 1 (2017), 86-98. https: //doi.org/10.1037/aca0000098

[5] Johannes Braumann and Sigrid Brell-Cokcan. 2011. Parametric robot control Integrated $\mathrm{CAD} / \mathrm{CAM}$ for architectural design. Integration Through Computation - Proceedings of the 31st Annual Conference of the Association for Computer Aided Design in Architecture, ACADIA 2011 (2011), 242-251.

[6] Johannes Braumann and Sigrid Brell Cokcan. 2012. Digital and physical tools for industrial robots in architecture: Robotic interaction and interfaces. International Journal of Architectural Computing 10, 4 (2012), 541-554. https://doi.org/10.1260/ 1478-0771.10.4.541

[7] H. L. Elshatarat, R. Biesenbach, M. Bani Younus, and T. A. Tutunji. 2016. MATLAB Toolbox implementation and interface for motion control of KUKA KR6R900-SIXX robotic manipulator. 16th International Conference on Research and Education in Mechatronics, REM 2015 - Proceedings (2016), 12-15. https: //doi.org/10.1109/REM.2015.7380367

[8] Behnaz Farahi. 2017. Material Behaviours in 3D-Printed Fashion Items. Architectural Design 87, 6 (2017), 84-91. https://doi.org/10.1002/ad.2242

[9] Matheus Gadelha, Subhransu Maji, and Rui Wang. 2018. 3D shape induction from 2D views of multiple objects. Proceedings - 2017 International Conference on 3D Vision, 3DV 2017 (2018), 402-411. https://doi.org/10.1109/3DV.2017.00053 arXiv: 1612.05872

[10] Madeline Gannon, Julián Sandoval, Kevyn McPhail, and Ben Snell. 2018. Manus - Exploring Pack Behaviors in Autonomous Robots - YouTube. Retrieved December 19, 2020 from https://www.youtube.com/watch?v=lyQwqi-Hycw $\{\&\}$ feature= emb\{\}\}title

[11] Emanuel Gollob. 2019. Doing Nothing with AI 1.0. Retrieved December 19, 2020 from https://www.emanuelgollob.com/doing-nothing-with-ai/

[12] Emanuel Gollob. 2019. Doing Nothing with AI 2.0. Retrieved December 19, 2020 from https://www.emanuelgollob.com/doing-nothing-with-ai-2/

[13] Emanuel Gollob. 2020. Shaky Savine \& Doing Nothing with AI. Retrieved December 19, 2020 from https://www.emanuelgollob.com/shaky-savine-doingnothing-with-ai/

[14] Ian J. Goodfellow, Jean Pouget-Abadie, Mehdi Mirza, Bing Xu, David WardeFarley, Sherjil Ozair, Aaron Courville, and Yoshua Bengio. 2014. Generative adversarial nets. Advances in Neural Information Processing Systems 3, January (2014), 2672-2680. arXiv:arXiv:1406.2661v1

[15] Michael D. Greicius, Ben Krasnow, Allan L. Reiss, and Vinod Menon. 2003. Functional connectivity in the resting brain: A network analysis of the default mode hypothesis. Proceedings of the National Academy of Sciences of the United States of America 100, 1 (2003), 253-258. https://doi.org/10.1073/pnas.0135058100

[16] Amirhossein Hajiagha Memar. 2019. Enhancing human-robot interaction with passive brain-computer interfaces: A neuroergonomic approach. Dissertation $A b$ stracts International: Section B: The Sciences and Engineering 80, 2-B(E) (2019), NoSpecified. http://ovidsp.ovid.com/ovidweb.cgi?T $=J S\{\&\} P A G E=$ reference $\{\&\} \mathrm{D}=$ psyc $16\{\&\} N E W S=N\{\&\} A N=2018-58621-159$

[17] John E. Harding and Paul Shepherd. 2017. Meta-Parametric Design. Design Studies 52 (2017), 73-95. https://doi.org/10.1016/j.destud.2016.09.005

[18] Kristina Höök. 2008. Affective loop experiences - What are they? Lecture Notes in Computer Science (including subseries Lecture Notes in Artificial Intelligence and Lecture Notes in Bioinformatics) 5033 LNCS (2008), 1-12. https://doi.org/10.1007/ 978-3-540-68504-3-1

[19] Natasha Kovacevic, Petra Ritter, William Tays, Sylvain Moreno, and Anthony Randal McIntosh. 2015. 'My virtual dream': Collective neurofeedback in an immersive art environment. PLOS ONE 10, 7 (2015), 1-18. https://doi.org/10.1371/journal. pone. 0130129

[20] Helmut Leder. 2003. Familiar and Fluent! Style-Related Processing Hypotheses in Aesthetic Appreciation. Empirical Studies of the Arts 21, 2 (2003), 165-175. https://doi.org/10.2190/g6mk-6kl7-ketb-elnd

[21] Helmut Leder, Benno Belke, Andries Oeberst, and Dorothee Augustin. 2004. A model of aesthetic appreciation and aesthetic judgments. British fournal of Psychology 95, 4 (2004), 489-508. https://doi.org/10.1348/0007126042369811

[22] Helmut Leder and Marcos Nadal. 2014. Ten years of a model of aesthetic appreciation and aesthetic judgments: The aesthetic episode - Developments and challenges in empirical aesthetics. British fournal of Psychology 105, 4 (2014), 443-464. https://doi.org/10.1111/bjop.12084

[23] Golan Levin, Lawrence Hayhurst, Steven Benders, and Fannie White. 2008. Double-Taker (Snout) - Interactive Art by Golan Levin and Collaborators. Retrieved December 19, 2020 from http://www.flong.com/archive/projects/snout/ index.html

[24] Octavio Loyola-Gonzalez. 2019. Black-box vs. White-Box: Understanding their advantages and weaknesses from a practical point of view. IEEE Access 7 (2019), 154096-154113. https://doi.org/10.1109/ACCESS.2019.2949286

[25] Mohammad Mohammadhassani, Hossein Nezamabadi-Pour, Mohd Zamin Jumaat, Mohammed Jameel, and Arul M.S. Arumugam. 2013. Application of artificial neural networks (ANNs) and linear regressions (LR) to predict the deflection of concrete deep beams. Computers and Concrete 11, 3 (2013), 237-252. https: //doi.org/10.12989/cac.2013.11.3.237

[26] Irene Neuner, Jorge Arrubla, Cornelius J. Werner, Konrad Hitz, Frank Boers, Wolfram Kawohl, and N. Jon Shah. 2014. The default mode network and EEG regional spectral power: A simultaneous fMRI-EEG study. PLoS ONE 9, 2 (2014). https://doi.org/10.1371/journal.pone.0088214

[27] M.D. Obermeyer, Ziad and Ph.D. Emanuel, Ezekiel J., M.D. 2016. Predicting the Future - Big Data, Machine Learning, and Clinical Medicine. New England fournal of Medicine 375, 13 (2016), 1212-1216. https://doi.org/10.1056/NEJMp1609300

[28] Marcus T. Pearce, Dahlia W. Zaidel, Oshin Vartanian, Martin Skov, Helmut Leder, Anjan Chatterjee, and Marcos Nadal. 2016. Neuroaesthetics: The Cognitive Neuroscience of Aesthetic Experience. Perspectives on Psychological Science 11, 2 (2016), 265-279. https://doi.org/10.1177/1745691615621274

[29] Matthew Pelowski, M. Forster, Pablo P.L. Tinio, Maria Scholl, and Helmut Leder. 2017. Beyond the lab: An examination of key factors influencing interaction with 'Real' and museum-based art. Psychology of Aesthetics, Creativity, and the Arts 11, 
3 (2017), 245-264. https://doi.org/10.1037/aca0000141

[30] Matthew Pike, Richard Ramchurn, Steve Benford, and Max L. Wilson. 2016 \#Scanners: Exploring the control of adaptive films using brain-computer interaction. Conference on Human Factors in Computing Systems - Proceedings 0 (2016), 5385-5396. https://doi.org/10.1145/2858036.2858276

[31] Anja Roye, Lea Höfel, and Thomas Jacobsen. 2008. Aesthetics of faces: Behavioral and electrophysiological indices of evaluative and descriptive judgment processes. Journal of Psychophysiology 22, 1 (2008), 41-57. https://doi.org/10.1027/0269-
8803.22.1.41

[32] Jiajun Wu, Chengkai Zhang, Tianfan Xue, William T. Freeman, and Joshua B. Tenenbaum. 2016. Learning a probabilistic latent space of object shapes via 3D generative-adversarial modeling. Advances in Neural Information Processing Systems Nips (2016), 82-90. arXiv:1610.07584

[33] Xin Yi, Ekta Walia, and Paul Babyn. 2019. Generative adversarial network in medical imaging: A review. Medical Image Analysis 58 (2019). https://doi.org/10. 1016/j.media.2019.101552 arXiv:1809.07294 